\title{
Article
}

\section{Would adults with autism be less likely to bury the survivors? An eye movement study of anomalous text reading}

Au Yeung, Sheena, Kaakinen, J.K., Liversedge, Simon Paul and Benson, Valerie

Available at http://clok.uclan.ac.uk/22319/

Au Yeung, Sheena, Kaakinen, J.K., Liversedge, Simon Paul ORCID: 0000-00028579-8546 and Benson, Valerie ORCID: 0000-0002-0351-4563 (2018) Would adults with autism be less likely to bury the survivors? An eye movement study of anomalous text reading. The Quarterly Journal of Experimental Psychology, 71 (1). pp. 280-290. ISSN 1747-0218

It is advisable to refer to the publisher's version if you intend to cite from the work. http://dx.doi.org/10.1080/17470218.2017.1322621

For more information about UCLan's research in this area go to http://www.uclan.ac.uk/researchgroups/ and search for <name of research Group>.

For information about Research generally at UCLan please go to http://www.uclan.ac.uk/research/

All outputs in CLoK are protected by Intellectual Property Rights law, including Copyright law. Copyright, IPR and Moral Rights for the works on this site are retained by the individual authors and/or other copyright owners. Terms and conditions for use of this material are defined in the policies page.

\section{CLoK}

Central Lancashire online Knowledge www.clok.uclan.ac.uk

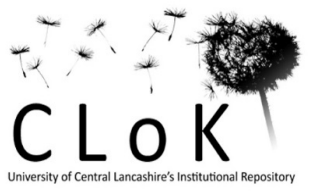




\title{
The Quarterly Journal of Experimental Psychology
}

\section{Would Adults with Autism be less likely to Bury the Survivors? An Eye Movement Study of Anomalous Text Reading}

\author{
Sheena K. Au-Yeung, Johanna K. Kaakinen, Simon P. Liversedge \& Valerie \\ Benson
}

To cite this article: Sheena K. Au-Yeung, Johanna K. Kaakinen, Simon P. Liversedge \& Valerie Benson (2017): Would Adults with Autism be less likely to Bury the Survivors? An Eye Movement Study of Anomalous Text Reading, The Quarterly Journal of Experimental Psychology, DOI: 10.1080/17470218.2017.1322621

To link to this article: http://dx.doi.org/10.1080/17470218.2017.1322621

View supplementary material $\sqsubset$

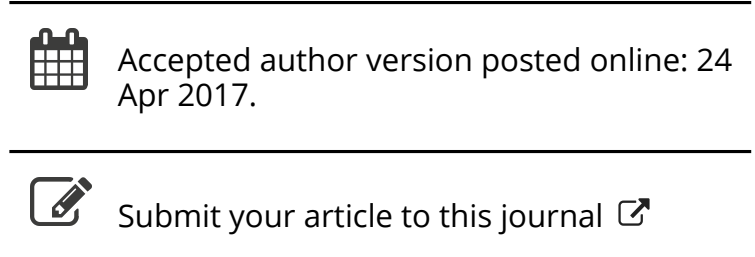

III Article views: 19

Q View related articles $\sqsubset$

View Crossmark data $\nearrow$ 
Publisher: Taylor \& Francis \& The Experimental Psychology Society

Journal: The Quarterly Journal of Experimental Psychology

DOI: $10.1080 / 17470218.2017 .1322621$

Running head: Anomaly Detection in ASD

Would Adults with Autism be less likely to Bury the Survivors? An Eye Movement Study of Anomalous Text Reading

Sheena K. Au-Yeung ${ }^{1}$, Johanna K. Kaakinen ${ }^{2}$, Simon P. Liversedge ${ }^{1}$, Valerie Benson ${ }^{1}$ ${ }^{1}$ School of Psychology, University of Southampton, UK

${ }^{2}$ Department of Psychology, University of Turku, Finland

Corresponding Author: Valerie Benson

Psychology

Shackleton Building (44)

Highfield Campus

University of Southampton

SO17 1BJ

e-mail: vb1@soton.ac.uk

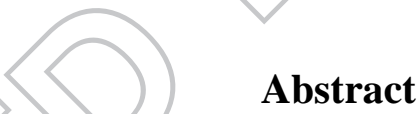

In a single eye movement experiment we investigated the effects of context on the time course of local and global anomaly processing during reading in adults with Autism Spectrum Disorder (ASD). In one condition short paragraph texts contained anomalous target words. Detection of the anomaly was only possible through evaluation of word meaning in relation to the global context of the whole paragraph (Passage Level Anomalies). In another condition the anomaly could be detected via computation of a local thematic violation within a single sentence embedded in the paragraph (Sentence Level Anomalies).

For the sentence level anomalies the ASD group, in contrast with the typically developing (TD) group, showed early detection of the anomaly as indexed by regressive eye movements from the critical target word upon fixation. Conversely, for the passage level anomalies, and in contrast with the ASD group, the TD group 
showed early detection of the anomaly, with increased regressive eye movements once the critical word had been fixated.

The reversal of the pattern of regression path data for the two groups, for the sentence and passage level anomalies, is discussed in relation to cognitive accounts of ASD.

Keywords: Autism, Eye movements, Reading, Anomaly, Context

\section{Introduction}

Autism Spectrum Disorder (ASD) is a lifelong neurodevelopmental condition characterised by persistent deficits in social communication and interaction, and restricted, repetitive patterns of behaviours, interests and activities (APA, 2013). Prevalence in the UK approximates to 10 per 1000 (Brugha et al., 2001), and the UK National Audit office has recently called for more research into adults with ASD (Clark, Scharaschkin, \& Xu, 2009), who may present as 'typical' on paper and pencil tests and have IQ within the normal range, but are nevertheless unable to lead independent lives.

ASD reflects the interaction of genetic, epigenetic, and environmental factors (Eapen, 2011). These factors contribute to pathological development in neural mechanisms, leading to structural and functional abnormalities of the brain and it has been suggested that it is these developing brain abnormalities that result in cognitive processing differences that translate into the behavioural syndrome known as ASD (Minshew, Williams, \& McFadden, 2008). How such translations occur, at present remains unknown.

What is known is that the language processing domain is one of the most obvious areas where cognitive differences manifest in ASD (e.g., Rapin \& Dunn, 2003; Tager-Flusberg, 1981). The current study therefore aimed to investigate cognitive processing during reading in adults with ASD without intellectual disabilities. Specifically, we examined whether there are differences in detection and processing for two different anomaly categories in ASD. One category of anomaly can be detected when reading a single sentence in isolation within a passage. The other category of anomaly can only be detected if the global context of the passage is processed during reading.

Reading ability in ASD has been reported to be highly variable (Nation, Clarke, 
Wright, \& Williams, 2006), but, readers with high functioning ASD have frequently been found to have intact performance for 'low-level' reading tasks, e.g., word decoding and identification (Brown, Oram-Cardy, \& Johnson 2013; Frith \& Snowling, 1983; Minshew, Goldstein, \& Siegel, 1995; Mayes \& Calhoun, 2006; Saldana, Carreiras, \& Frith, 2009) with significant impairments for 'higher-order' linguistic tasks, for example, for inferencing tasks (Minshew et al., 1995; Joliffe \& Baron-Cohen, 2000; Nation et al., 2006; Norbury \& Nation, 2011), and for the use of figurative language (Happé, 1995; Norbury, 2004). Passage comprehension has also been shown to be impaired in ASD (Minshew et al., 1995; Nation et al., 2006; Newman et al., 2007; Jones et al., 2009; but see also Brown et al., 2013).

In the Disordered Complex Information Processing (DCIP) model of ASD (Minshew \& Goldstein, 1988) ASD is presented as a neurological disorder stemming from under-connectivity between neocortical brain areas necessary for higher level processing, and over-connectivity in brain regions involved in lower-level processing, resulting in intact or superior performance for low level domain specific tasks (Just, Cherkassy, Keller, \& Minshew, 2004; Minshew et al., 2008), with deficits in high level cognitive processing tasks. In support of this theory similarities in performance, either in patterns of abilities and patterns of deficits have been reported in children with ASD (Williams, Goldstein, \& Minshew 2006) for a range of tasks, and an eye movement study in adults with ASD has reported evidence to show that equivalent performance outcomes can be underpinned by differences in processing for 'complex', but not 'simple' information (Benson, Castelhano, Au-Yeung, \& Rayner, 2012).

In relation to reading, according to the DCIP theory, the cognitive processes required to complete basic, comparatively low level, reading (e.g., word identification, initial syntactic parsing) would be intact in ASD. In contrast, more complex processes such as the computation of sentential meaning and its integration into the discourse representation should be impaired in ASD as a result of the requirement to access and process (often implicit) world knowledge. Also, cognitive load would be predicted to modulate performance.

An alternative theory, Weak Central Coherence (WCC) theory, proposes that individuals with ASD process information less efficiently in context than typically 
developing (TD) controls because they engage in piecemeal processing of local information (Frith, 1989; Happé, 1999). At the neurological level, WCC, in line with the DCIP theory, can also be explained by frontal-posterior underconnectivity (Frith, 2012; Just, Keller, \& Kana, 2013). A detail focused processing style in favour of local information processing in ASD has been demonstrated in cognitive tasks using visual and verbal stimuli (Booth \& Happé, 2010; Plaisted, Swettenham, \& Rees, 1999), and problems with detecting anomalies have been found in scene perception tasks in ASD (Joliffe \& Baron-Cohen, 2001; Benson et al., 2012).

In WCC theory text would be predicted to be read and understood in a less integrated way (Happé, 1999), with less effective linkage of words and sentences with the context and a more general failure to integrate information across a discourse. Such performance could be argued to reflect a failure to process text globally. However, several studies have found no evidence for impairments in the use of context in ASD, either for reading or for other language tasks, and for different paradigms investigating cognitive processing in ASD. For example, equivalent performance has been reported in ASD during semantic priming tasks (Hala, Pexman, \& Glenwright, 2007; Henderson, Clarke, \& Snowling, 2011; Norbury, 2005), and for a visual world paradigm (Brock, Norbury, Einav, \& Nation, 2008), and no evidence for impairments were noted in typical readers with subclinical autistic traits in a study that examined eye movements during a reading task (Caruana \& Brock, 2014). These studies demonstrate that although WCC stipulates impairments in the use of contextual information, such impairments have not always been observed. We note also that striving for global coherence is characteristic of typical readers.

For typical readers, a classic study by Barton and Sanford (1993) demonstrated that the global goodness of fit of a word to a whole passage context influences anomaly detection in text reading. In this study participants read a short text about an aeroplane crash followed by a question asking where should the survivors be buried. It was found that the overall detection rate for the anomaly (i.e., survivors are not buried) was only $30 \%$. These findings were replicated with new materials (Hannon \& Daneman, 2004), and the better the fit of the anomalous term to expectations based on the global context of the text, the more likely readers were to engage in shallow processing of the anomaly, and thus, the less likely to detect the anomaly. This is consistent with the idea of central coherence (Frith, 1989) that proposes that TD individuals strive to incorporate incoming information in its context 
to maximise meaningful coherence in the discourse representation, and this is achieved at the expense of processing local detailed information.

It is important to note that the majority of studies reported above that investigated different aspects of reading in ASD used accuracy or reaction times as the main performance measures. Very few studies have investigated eye movements during reading in ASD (Balestra et al., 2010, Bills, 2009). Thus little is known about on-line processing during anomaly detection in reading in ASD. However, an investigation of the time-course of TD individuals' processing of text containing anomalous material (Daneman, Lennertz, \& Hannon, 2007) revealed that detection of the anomaly was not immediate at the first encounter of the target word, but occurred later with more time spent looking back at the target words in the anomalous condition compared to the non-anomalous condition. No studies to date have used eye movement methodology to investigate on-line anomaly detection in text reading in ASD. Measurement of eye movements during reading provides information about both the nature and time course of linguistic processing (Liversedge \& Findlay, 2000; Rayner, 1998, 2009). Eye movements therefore have potential to inform in relation to on-line processing differences that exist between TD and ASD readers (sometimes referred to as differing processing styles; Frith \& Happé, 1994).

The aim of the current study was to investigate whether there were time course differences in how individuals with ASD detect and process anomalies in text comprehension, in comparison to a TD control group. We used modified stimuli from previous studies with TD readers (Barton \& Sanford, 1993; Daneman et al., 2007; Hannon \& Daneman, 2004) and created new stimuli to test hypotheses derived from two converging theories, WCC (Frith, 1989; 2012; Happé, 1999) and DCIP (Minshew \& Goldstein, 1988). Two different types of anomalies were devised: For paragraph level anomalies detection of the anomaly is dependent on global processing of the preceding passage context. For sentence level anomalies detection of the anomaly is independent of the preceding passage context (see Figure 1 for example stimuli). In order to detect a global (paragraph level) anomaly, the reader has to keep the whole of the preceding passage in mind and must integrate the meaning of the current sentence with that of the preceding context. In contrast, a local (sentence level) anomaly can be detected by reading a single sentence in isolation. Consequently, WCC theory predicts a failure to detect either type of anomaly if there are integration difficulties 
both within and between sentences in a passage in the ASD group. DCIP theory, in contrast, predicts that the cognitive load associated with the detection of passage level anomalies would make these more complex to process than the single sentence level anomalies. Increased memory load and an extended period of linguistic processing produce this load. As such, sentence level anomalies might be detected with more ease than the passage level anomalies in ASD. For the TD group, assuming that they are striving for global coherence in the discourse representation, then they should show the opposite pattern of effects, that is, early detection of passage level anomalies in comparison to sentence level anomalies. Increased reading times followed by regressions should reflect anomaly detection and efforts towards coherence checking and anomaly resolution.

\section{Method}

\section{Participants}

Participants with ASD were clinically diagnosed in the UK prior to the experiment and were required to provide formal evidence of their diagnosis in order to be included in the study. Participants were recruited on a voluntary basis from the National Autistic Society, Children on the Autism Spectrum Parents' Association, and also from a database of volunteers who had previously taken part in other studies, either recruited from the general public or from the University of Southampton through word of mouth or online adverts. The TD group were recruited from the University of Southampton or from the general population through word of mouth. Thirty-eight volunteers participated in the study; 20 in the TD group and 18 in the ASD group. Two ASD, and one TD participant were excluded for scoring lower than 90 on at least one of the IQ subsets. One ASD participant was excluded for failing to supply evidence of formal diagnosis. Three TD participants were excluded due to unsatisfactory calibration. The final sample included 15 ASD and 16 TD participants who were group-matched on verbal, performance and full scale IQ as estimated by the Weschler Abbreviated Scale of Intelligence (WASI; The Psychological Corporation, 1999). Comparing ASD and TD adults who fall within the normal IQ range allows the identification of cognitive features that are unique to ASD and which are not attributable to intellectual disability (Minshew \& Williams, 2008). The ASD group had a slightly greater mean age than the TD group due to five participants being in the 40- 
50 year (four participants) and 50-60 year (one participant) age range, and this elevated ASD mean was also a result of one of the TD participants with a higher age (49) being excluded from the analyses for failing to meet one of the criteria. Participant characteristics are presented in Table 1. Although age could potentially be a confound, we have reason to believe that this has not affected the results for the current study. Whilst previous research has shown that eye movements during reading were affected by age (e.g., Paterson, McGowan, \& Jordan, 2013), these differences were found in much older adults (age 65+). It was found that older adults took longer to read, make more and longer fixations, and make more regressions compared to younger adults (age 18 to 30) (but note that both age groups had good comprehension). None of the participants in the current study are within the age range of older adults as classified by previous eye movement research. The ASD group scored significantly higher on the Autism Spectrum Quotient (AQ; Baron-Cohen, Wheelwright, Skinner, Martin, \& Clubley, 2001) demonstrating greater levels of self-reported autistic traits.

\section{[Insert Table 1 here]}

\section{Materials}

Twenty four passages of text (some adopted from previous studies; Barton \& Sanford, 1993; Daneman et al., 2007; Hannon \& Daneman, 2004), consisting of five to seven sentences were created for the stimuli. For 12 of the passages (Passage Anomalies), the anomalous target word was inconsistent with the preceding passage context, but was not anomalous when the sentence containing the target word was read in isolation. For the other 12 passages (Sentence Anomalies), the anomalous target word was designed to have a global good fit with the context of the passage (e.g., the word survivors fits well in the context of an aeroplane crash) but was anomalous when the sentence containing it was read in isolation (ordinarily survivors are not buried). Baseline non-anomalous versions of each passage were also created. See Figure 1 for examples, and Appendix 1 in the Supplemental Material for the full stimulus set.

Target words in the anomalous and non-anomalous condition were also closely matched in word length [Anomalous: $M=6.92, S D=2.34$, Non-anomalous: $M=$ 7.17, $S D=2.75, t(46)=.340, p=.736$ ], and frequency (counts per million words) 
[Anomalous: $M=92.92, S D=294$, Non-anomalous: $M=100, S D=222, t(46)=$ $.097, p=.923$ ], as taken from the CELEX corpus (Max Planck Institute for Psycholinguistics, n. d.).

Four stimuli lists were created, each consisting of 24 passages that included 12 anomalous text passages and 12 non-anomalous text passages. Within the 12 anomalous passages, six of those were sentence anomalies and the other 6 were passage anomalies. For the 12 non-anomalous passages, six of those were control passages for the sentence anomalies and six were control passages for the passage anomalies. Stimuli were rotated across lists according to a Latin Square. Participants viewed all 24 passages in a stimuli list, but only one of the two versions of a passage appeared in any one stimuli list, therefore participants were presented with either the anomalous or the non-anomalous version of the same passage. Passages were randomly presented for each participant on a 1024 x 768 monitor, in black, size 14 Courier New font, with equal character spacing, on a white background. Triple spacing facilitated recording of eye movements in different lines of text.

\section{Eye movement recording}

Participants viewed the stimuli binocularly and eye movements were recorded for the right eye using an Eyelink 1000 eye tracker (SR Research Ltd, Osgoode, Canada) with a viewing distance of $70 \mathrm{~cm}$. Head position was stabilised by a chin and forehead rest. Participants were calibrated using a nine-point matrix that covered the dimensions of the display screen.

\section{Design}

The experiment was a mixed design with two within-participant variables Anomaly Type (Passage (Global) vs Sentence (Local) anomalies), Target Word (Anomalous ys Non-anomalous target words), and a between-participant variable Group (TD vs ASD).

\section{Procedure}

Participants were instructed to read the short stories carefully. Following calibration, the text passages were presented one at a time and participants pressed a game pad button when they had finished reading to initiate presentation of a question. Two questions about factual content of the passage, unrelated to the anomalies, required forced-choice yes-no answers on every trial, and were designed to inhibit participants from actively looking for anomalies.

\section{Statistical Analyses}


Statistical analyses were performed using (generalized) linear mixed effects models using the lme4 package (Version 1.1-12; Bates, Maechler, Bolker, \& Walker, 2015) for R (version 3.3.1; R Core Team, 2016).

The text comprehension scores were analysed with a generalized linear mixed effects model (logit) with Anomaly Type (Sentence vs Passage), Word type (anomalous vs. non-anomalous), Participant group (TD vs. ASD) and all two- and three-way interaction terms as fixed factors; sum contrasts were used to code the factor levels. Random intercepts for participants and items were included in the random structure of the models. The model is presented in Appendix 2 of the Supplemental Material. Interactions (as indicated by $p<.10$ ) were followed up by fitting the model at different levels of anomaly type (by using dummy coding); the effects are reported in the text.

Analyses of the eye movement measures were conducted separately for sentence and passage anomaly texts and for each dependent measure by using linear mixed effects models. Observations that exceeded 2.5 SD of each participant's mean in the experimental condition would have been excluded from the analyses, but no observations met this criterion for any of the measures. Word type (anomalous vs. non-anomalous), participant group (TD vs. ASD) and their interaction term were entered into the models as fixed factors; sum contrasts were used to code the factor levels. Random intercepts for participants and items were included in the random part of the models. Observations for which residual $>2.5 S D$ were excluded as outliers, and the analysis was rerun with a trimmed dataset. $P$-values for fixed effects were estimated using the Satterthwaite estimation for degrees of freedom available in the lmerTest package (Kuznetsova, Brockhoff, \& Christensen, 2014). The anomaly effect within each group was tested by fitting the model at different levels of group (by using dummy coding) if the Word type * Group interaction term indicated an effect $(p<.10)$; these effects are reported in the text below. All models are presented in Appendix 2 of the Supplemental Material.

\section{Regions of Interest}

Each passage of text was auto-segmented into single word regions using Eyelink Dataviewer. Each punctuation mark that comes after a word was merged into the same single word region as that word. The empty spaces (the size of one letter) that are between words were included in the same single word region as the word that comes directly after the space. Each single word region was 80 pixels in height, leav- 
ing no space in between single word regions on different lines of text. Each single word region also touched the edge of the regions for the words next to it, leaving no space in between single word regions on the same line. The single word regions were then grouped with neighbouring regions, if necessary, to create the regions of interest, including the critical word region, the spill-over region and the passage end region (see Figure 1 Legend which details the regions used in the analyses).

\section{Results}

\section{Comprehension Task}

Descriptive statistics for comprehension accuracy are presented in Table 2. The results were analysed with a generalized linear mixed effects model (logit) with Anomaly Type (Sentence vs Passage), Target Word (Anomalous vs Normal), and Group (TD vs ASD) as fixed effects and Participant and Text as random factors. Comprehension scores were higher overall for the passage anomaly texts than for sentence anomaly texts, $b=.52, \mathrm{z}=4.79, p<.001$. Comprehension was poorer for texts that contained an anomaly than for normal texts, $b=-.32$, $\mathrm{z}=-4.77, p<.001$. An interaction between Anomaly Type and Target Word, $b=-.30, \mathrm{z}=-4.46, p<.001$, indicated that the difference in the comprehension scores between anomalous and normal texts was significant in the passage anomaly texts $(\mathrm{b}=-.61, \mathrm{z}=-5.89, \mathrm{p}<.001)$ but did not reach significance in the sentence anomaly texts $(b=-.02, z=-.25, p=.805)$. There was no effect of Group, nor any interactions with group, which indicated that any differences in eye movements reflect differences in on-line processing that is related to the experimental manipulations, rather than to any group differences in terms of overall text comprehension.

[Insert Table 2 here]

\section{Eye movement Results}

The following eye movement measures were computed for the ROIs: First pass reading time (gaze duration for single word regions) was defined as the summed duration of the fixations in a region until the reader moved their eyes to fixate another region; Regression path reading time was defined as the sum of all fixations from the first fixation in a region until the participant made a fixation to the right of that region. Regression path reading times, therefore, potentially included fixations in earlier regions that were made after regressions. Total reading time was defined as the sum of all fixations in a region. First-pass reading time gives an indication of early 
processing; regression path reading times also reflect early processing difficulty along with re-inspection of text in an effort to recover from difficulty; and total reading times provides a measure of overall processing difficulty (Rayner, 1998).

Data for the passage and sentence anomaly texts were analysed separately with linear mixed effects models using the lme4 package (Bates, Maechler, Bolker, \& Walker, 2015) for R (R Core Team, 2016). Word type (anomalous vs. nonanomalous) and participant group (TD vs. ASD) were entered into the models as fixed factors using sum contrasts. Random intercepts for participants and items were included in the random structure of the models. Models were trimmed by removing the highest-order term (starting from the two-way interaction) with the smallest $|\mathrm{t}|$ value and comparing the reduced model to the previous model using $\mathrm{X}^{2}$ likelihoodratio tests. $P$-values for fixed effects in the final models were estimated using the lmerTest package (Kuznetsova, Brockhoff, \& Christensen, 2014). The anomaly effect within each group was tested if the Word type by Group interaction term indicated an effect $(p<.10)$. Separate models were fitted for each dependent measure. Final models are presented in Appendix 2 of the Supplemental Material.

\section{Critical Word Region}

Sentence anomalies. Neither group showed an effect of sentence anomaly in gaze duration on the critical word, although the ASD reading times were numerically higher for anomalous versus control words than the TD reading times, see Table 3. The analysis of the regression path duration from the critical word, however, revealed a Word type by Group interaction, $b=76.71, t=3.09, p=.002$ and follow-up comparisons showed that readers with ASD demonstrated a sentence anomaly effect (i.e., longer regression path times on the anomalous vs. non-anomalous word), $b=149.67, t=4.07, p<.001$, whereas TD readers did not, $b=-3.75, t=-.11, p=.911$.

For the total reading time on the critical word there was an interaction between Word type and Group, $b=75.34, t=2.46, p=.015$, indicating that the anomaly effect was of different magnitude in the two groups. Readers with ASD also showed a sentence anomaly effect in the total fixation time, $b=211.00, t=4.753, p<.001$ whereas for TD readers the sentence anomaly effect failed to reach significance in the total fixation time, $b=60.31, t=1.43, p=.155$.

[Insert Table 3 here]

Passage anomalies. The analysis of the gaze duration on the critical word showed no indication of an interaction between group and word type. There was a 
robust passage anomaly effect across both participant groups, $b=16.32, t=2.65$, $p=.009$. However, the analysis of the regression path duration for the critical word revealed a marginal interaction between word type and group, $b=-47.51, t=-1.87$, $p=.063$. Follow-up comparisons showed that ASD readers did not show a passage anomaly effect in regression path times, $b=-19.52, t=-.50, p=.617$, whereas TD readers had longer regression path times for anomalous than non-anomalous words, $b=75.50, t=2.32, p=.021$, a pattern that is opposite to that shown in the sentence anomaly condition for the two groups. In the total fixation time on the critical word there was a robust passage anomaly effect across both participant groups, $b=86.38$, $t=6.09, p<.001$. Figure 2 shows the regression path data for both groups for both conditions.

[Insert Figure 2 about here]

\section{Spill-over Region}

Increased first-pass reading times on the word following a target word (i.e., spill-over region) are associated with processing difficulty in integrating the target word into the sentence context (Rayner, Warren, Juhasz, \& Liversedge, 2004). There were no effects of anomaly detection that spilled-over to the next word in the sentence (see Table 4 for descriptive statistics) showing that any processing difficulty that occurred at the target word did not persist through to fixations made on the next word in the sentence. This was the case for sentence and passage anomaly conditions.

\section{[Insert Table 4 here]}

\section{Passage End Region}

Regression path reading time for the last word of the passage was analysed to determine whether participants spent more time re-reading the passage in the anomalous condition once they had initially read through the text in its entirety.

Sentence anomalies. Participants with ASD showed longer overall regression path reading time from the last word of the passage than TD readers, irrespective of whether there was an anomaly present in the text or not, $b=726.07, t=2.69, p=.013$.

Passage anomalies. As with the sentence anomalies, for the passage anomalies participants with ASD showed longer overall regression path reading time from the last word of passage than TD readers, $b=755.23, t=2.85, p=.010$.

The regression path reading times for the passage end of both the sentence and passage anomalies indicate that the ASD readers engaged in significantly more re-reading of the passages than did the TD readers. 


\section{Discussion}

The aim of this study was to investigate time course differences in how individuals with ASD detect and process local and global anomalies in text comprehension. The ability to detect a passage level (global) anomaly in the present experiment depends on the ability to construct, maintain and use a representation of the meaning of the passage whilst reading subsequent sentences. In contrast, local anomalies could be detected on the basis of a semantic violation within a single sentence. Any preference to globally process the whole passage context could in fact impede performance for the sentence level anomaly condition, as has been reported previously (Barton \& Sanford, 1993).

For the sentence level anomalies the ASD group showed early detection of the anomaly, coupled with immediate attempts to check, and if possible, resolve the anomaly, as shown by regressive eye movements from the critical target word upon fixation. They also showed greater total time fixating anomalous compared to nonanomalous target words. In contrast, for the sentence level anomalies, the TD group showed no early anomaly detection, and therefore unsurprisingly, there was no evidence of an immediate attempt to try to resolve any anomaly once the anomalous word had been fixated. The TD group did detect the sentence anomaly, but later, as revealed by the total reading time measure. Thus efforts to maximise global coherence in the TD group explains why they failed to immediately regress to try to resolve the sentence level anomalies. A reduced sensitivity to the goodness of the global fit of the target word with the rest of the passage content in the ASD group offers an explanation as to why ASD readers detected the local anomaly more readily.

Conversely, for the passage anomaly condition the TD group showed early detection of the anomalies, followed by immediate attempts to resolve these, as indicated by increased regressive eye movements once the critical word had been fixated. There was also an effect of greater total time for the anomalous compared to the control target words in both groups for the passage anomalies. In contrast, although there was evidence from the first pass gaze duration measures that the ASD group showed early detection of the anomalies too, they did not immediately regress in order to resolve these, and attempts to do that were only apparent in the later processing measure of total fixation time. Thus the pattern of data for the two groups was reversed for the sentence and passage level anomalies. 
The WCC predicts that the ASD group would take longer to attempt to go back and resolve passage level anomalies because of a preference to focus on the details. In that respect the regression path data for the ASD group do support the WCC theory, if it is accepted that ASD have no problems integrating information at the single sentence level. However, because the ASD group also show on-line detection of the anomaly for the passage level condition, as reflected by the gaze duration and the total time fixating the target word - it is not the case that ASD readers are overall less sensitive to global context, they just take longer to process anomalies in that condition. The data suggest that the passage level anomalies are detected (encoded) in the same way in both groups, but an incomplete representation of these within the global passage context in the ASD group, could explain why they fail to regress immediately to try to resolve the anomaly in that condition. Also, the WCC theory would predict integration difficulties between words within sentences, and this was clearly not observed for the sentence level anomalies in the current experiment.

In sum the ASD participants clearly integrated words within the sentential interpretation, but appeared to be less effective at integrating the sentential meaning with the discourse representation, at least to the same degree as the TD group did in order to form a coherent globah representation of the whole passage. The DCIP theory predicts that as cognitive load increased ASD readers should experience difficulties with integration. In the current study it can be argued that there is an increased cognitive load associated with passage level anomaly detection since a detailed representation of the context had to be maintained in memory alongside concurrent linguistic processing. A representation of the meaning of context was not necessary in order to detect the sentence level anomalies. Thus, the failure to regress back into the text from first fixation on the target word, coupled with increased total times observed for the passage level anomaly condition in the ASD group provide some support for the DCIP theory.

In a recent study (Tirado \& Saldana, 2016) the amount of text intervening between sentences containing important linking information for text comprehension was manipulated and it was found that integration of information over longer passages of text was poorer in ASD relative to TD readers. This was observed as a reduction in accuracy for comprehension questions when there was more intervening text between relevant information and questions interrogating that information. This 
observation occurred even though ASD readers appeared to compute inferences online, as indexed by reading time measures. Overall the implication from this study is that inferred information may not be stored or integrated into the discourse representation as efficiently in ASD as in TD readers. One possible reason for this may be that ASD participants do not develop a fully specified and integrated discourse representation as they read, and this would explain why the ASD participants are faster to detect sentence, relative to passage, level anomalies in the current study.

An additional finding from the current study showed that ASD participants spent significantly greater time re-reading all types of passages after their initial reading, in comparison to the TD group. This finding is consistent with the results from a previous study investigating irony comprehension in ASD (Au-Yeung, Kaakinen, Liversedge, \& Benson, 2015) and the question of exactly why this effect emerges in ASD is clearly worthy of further empirical examination.

At this point we note a possible limitation to our conclusions. Although we included comprehension questions in our experiment to make sure that our participants were engaging in natural reading, all of our questions interrogated aspects of the text that were not relevant to the reader's interpretation of the anomaly. To be clear, our questions provided no indication as to whether readers had actually detected an anomaly, and if they had, what interpretation they formed of the anomalous text. Of course, this aspect of our experimental design was entirely purposeful to avoid drawing our participants' attention to the experimental manipulations but it does limit any claims we may make as to how readers did actually interpret the anomalous sentences. However, despite this limitation, it remains the case that our eye movement data provide very strong evidence that the anomalies were detected, and our comprehension data do indicate that readers were processing the sentences that they read normally.

In conclusion, detailed eye movement analyses have revealed subtle on-line processing differences that provide evidence for intact use of context in detecting anomalies in passage reading, but time course differences in efforts to resolve different types of anomaly in ASD. The initial fixation data show that the ASD group are as sensitive to the TD group for early detection of the passage anomalies, but they do not immediately try to resolve these. For the sentence anomalies the regression 
path data show that the ASD group immediately regress in an attempt to resolve the anomalies, whereas the TD group do this to a far lesser extent. The findings are partly in line with predictions derived from two converging cognitive theories of ASD, namely WCC and DCIP theory. More generally, differences in anomaly detection across passages and within individual sentences in ASD may arise from less efficient construction of a detailed representation of the discourse, indicating that, at least figuratively, the ASD participants would be less likely to 'bury the survivors'.

\section{Supplemental Material}

The Supplemental Material for this paper can be found at the address

\section{Ethics}

This study was approved by the University of Southampton Research Governance Office. Informed consent was provided by participants prior to participation in the study.

\section{Competing Interests}

The authors have no competing interests.

\section{Author Contributions}

SA designed the experiment, created the stimuli, collected the data, carried out some early analysis of the data, and contributed to the write up of the study JK carried out a substantial amount of the data analysis and wrote up the results. SL and VB contributed to the experimental design, advised on eye movement data analysis, and provided intellectual contributions to the interpretation of the data and VB drafted the manuscript. All authors gave final approval for publication.

\section{Funding}

This work was funded by the Economic and Social Research Council (ESRC Reference Number: ES/1019723/1 


\section{References}

American Psychiatric Association (2013). Diagnostic and statistical manual of mental disorders ( $5^{\text {th }}$ ed). Washington, DC: Author. doi: 10.1176/appi.books.9780890425596.744053

Au-Yeung, S. K., Kaakinen, J. K., Liversedge, S. P., \& Benson, V. (2015). Processing of written irony in Autism Spectrum Disorder: An eye-movement study. Autism Research, 8(6), 749-760. doi: 10.1002/aur.1490

Balestra, A., Boso, M., Orio, S., Pazzaglia, R., Ravarelli, A., \& Zanetti, M. A. (2010). Analyzing text comprehension deficits in autism with eye tracking: A case study. In 3rd Conference on Human System Interaction, p. 230-235. doi: 10.1109/HSI.2010.5514562

Baron-Cohen, S., Wheelwright, S., Skinner, R., Martin, J., \& Clubley, E. (2001). The Autism Spectrum Quotient (AQ): Evidence from Asperger syndrome/High functioning autism, males and females, scientists and mathematicians. Journal of Autism and Developmental Disorders, 31(1), 5-17. doi: 10.1023/A:1005653411471

Barton, S. B., \& Sanford, A. J. (1993). A case study of anomaly detection: Shallow semantic processing and cohesion establishment. Memory and Cognition, 21(4), 477 - 487. doi: 10.3758/BF03197179

Bates, D., Maechler, M., Bolker, B., \& Walker, S. (2015). Fitting Linear MixedEffects Models Using lme4. Journal of Statistical Software, 67(1), 148.doi:10.18637/jss.v067.i01.

Benson, V, Castelhano, M., Au-Yeung, S. K., \& Rayner, K. (2012). Eye movements reyeal no immediate "WOW" ("which one's weird") effect in autism spectrum disorder. Quarterly Journal of Experimental Psychology, 65(6), 1139-1150. doi: 10.1080/17470218.2011.644305

Bills, M. A. A. (2009). Central coherence in autism: A modified approach to studying homographs. Unpubished doctoral thesis . University of Utah, USA.

Booth, R., \& Happé, F. (2010). "Hunting with a knife and ... fork": Examining central coherence in autism, attention deficit/hyperactivity disorder, and typical development with a linguistic task. Journal of Experimental Child Psychology, 107(4), 377-393. doi: 10.1016/j.jecp.2010.06.003 
Brock, J., Norbury, C., Einav, S., \& Nation, K. (2008). Do individuals with autism process words in context? Evidence from language-mediated eye-movements. Cognition, 108(3), 896-904. doi: 10.1016/j.cognition.2008.06.007

Brown, H. M., Oram-Cardy, J., \& Johnson, A. (2013). A meta-analysis of the reading comprehension skills of individuals on the autism spectrum. Journal of Autism and Developmental Disorders, 43(4), 932-955. doi: 10.1007/s10803-012$1638-1$

Brugha, T. S., McManus, S., Bankart, J., Scott, F., Purdon, S., Smith, J., ... Meltzer, H. (2011). Epidemiology of autism spectrum disorders in adults in the community in England. Archives of General Psychiatry, 68(5), 459-465. doi:10.1001/archgenpsychiatry.2011.38

Caruana, N. \& Brock, J. (2014). No association between autistic traits and contextual influences on eye-movements during reading. PeerJ, 2, e466. doi: 10.7717\%2Fpeerj.466

Clark, F., Scharaschkin, A. \& Xu, D. (2009). Supporting autism through adulthood. Norwich, UK: National Audit Office. TSO (The Stationary office).

Daneman, M., Lennertz, T., \& Hannon, B. (2007). Shallow semantic processing of text: Evidence from eye movements. Language and Cognitive Processes, 22(1), 85-105. doi: 10,1080/01690960500372725

Eapen, V. (2011). Genetic basis of autism: is there a way forward? Current Opinion Psychiatry, 24(3): 226 -236. doi: 10.1097/YCO.0b013e328345927e

Frith, U. (1989). Autism. Explaining the enigma. Oxford, England: Basil Blackwell.

Frith, U. (2012) Why we need cognitive explanations of autism. Quarterly Journal of Experimental Psychology. 65(11), 2073-2092. doi: 10.1080/17470218.2012.697178

Frith, U., \& Happé, F. G. E. (1994). Autism: beyond "theory of mind" . Cognition, 50(1-3), 115-132. doi: 10.1016/0010-0277(94)90024-8

Frith, U., \& Snowling, M. (1983). Reading for meaning and reading for sound in autistic and dyslexic children. British Journal of Developmental Psychology, 1(4), 329-342. doi: j.2044-835X.1983.tb00906.x

Hala, S., Pexman, P. M., \& Glenwright, M. (2007). Priming the meaning of homographs in typically developing children and children with autism. Journal of Autism and Developmental Disorders, 37(2), 329-340. doi: 10.1007/s10803006-0162-6 
Hannon, B., \& Daneman, M. (2004). Shallow semantic processing of text: An individual- differences account. Discourse Processes, 37(3), 187-204. doi: 10.1207/s15326950dp3703_1

Happé, F. G. E. (1995). Understanding minds and metaphors - insights from the study of figurative language in autism. Metaphor and Symbolic Activity, 10(4), 275295. doi: 10.1207/s15327868ms1004_3

Happé, F. G. E. (1999). Autism: Cognitive deficit or cognitive style? Trends in Cognitive Sciences, 3(6), 216-222. doi: 10.1016/S1364-6613(99)01318-2

Henderson, L. M., Clarke, P. J., \& Snowling, M. J. (2011). Accessing and selecting word meaning in autism spectrum disorder. Journal of Child Psychology and Psychiatry, 52(9), 964-973. doi: 10.1111/j.1469-7610.2011.02393.x

Jolliffe, T., \& Baron-Cohen, S. (2000). Linguistic processing in high-functioning adults with autism or Asperger's syndrome. Is global coherence impaired? Psychological Medicine, 30(5), 1169-1187. doi: 10.1017/s003329179900241x

Joliffe, T., \& Baron-Cohen, S. (2001). A test of central coherence theory: Can adults with high-functioning autism or Asperger syndrome integrate objects in context? Visual Cognition, 8(1), 67-101. doi: 10.1080/13506280042000036

Jones, C. R. G., Happe, F., Golden, H., Marsden, A. J. S., Tregay, J., Simonoff, E., . . . Charman, T. (2009). Reading and Arithmetic in Adolescents With Autism Spectrum Disorders. Peaks and Dips in Attainment. Neuropsychology, 23(6), 718-728. doi: 10.1037/a0016360

Just, M. A., Cherkassky, V.L., Keller, T. A., \& Minshew, N. J. (2004). Cortical activation and synchronization during sentence comprehension in highfunctioning autism: evidence of underconnectivity. Brain, 127(8), 1811-1821. doi: 10.1093/brain/awh199

Just, M. A., Keller, T. A., \& Kana, R. K. (2013). A theory of autism based on frontalposterior underconnectivity. In M. A. Just \& K. A. Pelphrey (Eds.), Development and brain systems in autism (pp. 35-63). New York: Psychology Press.

Kuznetsova, A., Brockhoff, P. B., \& Christensen, R. H. B. (2014). lmerTest: Tests for random and fixed effects for linear mixed effect models (lmer objects of lme4 package). R package version 2.0-3. Retrieved from http://CRAN.Rproject.org/package=lmerTest 
Liversedge, S. P., \& Findlay, J. M. (2000). Saccadic eye movements and cognition. Trends in Cognitive Science, 4(1), 6-14. doi: 10.1016/S1364-6613(99)01418-7

Max Planck Institute for Psycholinguistics (n. d.). WebCelex. Retrieved from http://celex.mpi.nl/

Mayes, S. D., \& Calhoun, S. L. (2006). Frequency of reading, math, and writing disabilities in children with clinical disorders. Learning and Individual Differences, 16(2), 145-157. doi: 10.1016/j.lindif.2005.07.004

Minshew, N. J., \& Goldstein, G (1988). Autism as a disorder of complex information processing. Mental Retardation and Developmental Disabilities Research Reviews, 4(2): 129-136. doi: 10.1002/(SICI)1098-2779(1998)4:2<129:AIDMRDD10>3.0.CO;2-X

Minshew, N. J., Goldstein, G., \& Siegel, D. J. (1995). Speech And Language In HighFunctioning Autistic Individuals. Neuropsychology, 9(2), 255-261. doi: 10.1037//0894-4105.9.2.255

Minshew, N. J., \& Williams, D. L. (2008). Brain behavior connections in autism. Speaker's Journal, 8.5, 25 - 43. Retrieved from

http://thetransitionteam.weebly.com/uploads/9/1/2/5/9125478/behavior_conne ctions_in_austism.pdf

Minshew, N. J., Williams, D. L., \& McFadden, K. (2008). Information processing, neural connectivity, and neuronal organization. In A. W. Zimmerman (Ed.), Autism: Current Clinical Neurology (pp. 381-405). Totowa, NJ: Humana Press.

Nation, K., Clarke, P., Wright, B., \& Williams, C. (2006). Patterns of reading ability in children with autism spectrum disorder. Journal of Autism and Deyelopmental Disorders, 36(7), 911-919. doi: 10.1007/s10803-006-0130-1

Newman, T. M., Macomber, D., Naples, A. J., Babitz, T., Volkmar, F., \& Grigorenko, E. L. (2007). Hyperlexia in children with Autism Spectrum Disorders. Journal of Autism and Developmental Disorders, 37(4), 760-774. doi: 10.1007/s10803-006-0206-y

Norbury, C. F. (2004). Factors supporting idiom comprehension in children with communication disorders. Journal of Speech Language and Hearing Research, 47(5), 1179-1193. doi: 10.1044/1092-4388(2004/87)

Norbury, C. F. (2005). Barking up the wrong tree? Lexical ambiguity resolution in children with language impairments and autistic spectrum disorders. Journal 
of Experimental Child Psychology, 90(2), 142-171. doi:

10.1016/j.jecp.2004.11.003

Norbury, C., \& Nation, K. (2011). Understanding Variability in Reading

Comprehension in Adolescents With Autism Spectrum Disorders: Interactions With Language Status and Decoding Skill. Scientific Studies of Reading, 15(3), 191-210. doi: 10.1080/10888431003623553

Paterson, K. B., McGowan, V. A., \& Jordan, T. R. (2013). Effects of adult aging on reading filtered text: evidence from eye movements. PeerJ, 1, e63. doi: 10.7717/peerj.63

Plaisted, K., Swettenham, J., \& Rees, L. (1999). Children with autism show local precedence in a divided attention task and global precedence in a selective attention task. The Journal of Child Psychology and Psychiatry and Allied Disciplines, 40(5), 733-742. doi: 10.1111/1469-7610.00489

Rapin, I., \& Dunn, M. (2003). Update on the language disorders of individuals on the autistic spectrum. Brain and Development, 25(3), 166-172. doi: 10.1016/S0387-7604(02)00191-2

Rayner, K. (1998). Eye movements in reading and information processing: 20 years of research. Psychological Bulletin, 124(3), 372-422. doi: 10.1037//00332909.124.3.372

Rayner, K. (2009). Eye movements and attention in reading, scene perception, and visual search. Quarterly Journal of Experimental Psychology, 62(8), 1457 1506. doi: $10.1080 / 17470210902816461$

Rayner, K., Warren, T., Juhasz, B. J., \& Liversedge, S. P. (2004). The effect of plausibility on eye movements in reading. The Journal of Experimental Psychology: Learning, Memory, and Cognition, 30(6), 1290-1301. doi: 10.1037/0278-7393.30.6.1290

R Core Team (2016). R: A language and environment for statistical computing. R Foundation for Statistical Computing, Vienna, Austria. Retrieved from http://www.R-project.org/ version 3.3.1

Saldana, D., Carreiras, M., \& Frith, U. (2009). Orthographic and phonological pathways in hyperlexic readers with autism spectrum disorders. Developmental Neuropsychology, 34(3), 240-253. doi: 10.1080/87565640902805701 
Tager-Flusberg, H. (1981). On the nature of linguistic functioning in early infantileautism. Journal of Autism and Developmental Disorders, 11(1), 45-56. doi: 10.1007/bf01531340

The Psychological Corporation (1999). Wechsler Abbreviated Scale of Intelligence (WASI) Manual. San Antonio, TX: The Psychological Corporation.

Tirado, M. J., \& Saldana, D. (2016). Readers with autism can produce inferences, but they cannot answer inferential questions. Journal of Autism and Developmental Disorders, 46(3), 1025-1037. doi: 10.1007/s10803-015-2648-6

Williams, D. L., Goldstein, G., \& Minshew, N. J. (2006). Neuropsychologic functioning in children with autism: Further evidence for disordered complex information processing. Child Neuropsychology, 12(4-5), 279-298. doi: $10.1080 / 09297040600681190$

\section{Figure Captions}

Figure 1. Panel (a) shows an example of a Passage Level Text (global context). Panel (b) shows an example of a Sentence Level Text (local context). The Regions of Interest are numbered and underlined for each type of text respectively. In Panel (a) the critical word (1) is "Japanese" for the anomalous version of the passage or "Chinese" for the non-anomalous version of the passage. In Panel (b) the critical word (1) is "survivors" for the anomalous version of the passage or "dead" for the non-anomalous version of the above passage. The spill-over region (2) includes the next progressive word in the passage after the critical word, however, if that word is a function word then the spillover region will extend to include the next progressive word in the passage. The passage end region (3) is the final word in the passage.

Figure 2. Regression path durations (ms) for the Critical Word Region for sentence and passage anomalies for both groups. 
Table 1.

Participant Characteristics

\begin{tabular}{|c|c|c|c|c|c|c|c|c|}
\hline \multirow[b]{2}{*}{ Measure } & \multicolumn{3}{|c|}{ TD } & \multicolumn{3}{|c|}{ ASD } & \multirow[b]{2}{*}{$t$} & \multirow[b]{2}{*}{$P$} \\
\hline & $M$ & $S D$ & Range & $M$ & $S D$ & Range & & \\
\hline Age & 24.6 & 5.8 & $18-35$ & 32.2 & 11.4 & $20-52$ & 2.592 & .017 \\
\hline Verbal IQ & 116 & 9.9 & $95-138$ & 119 & 12.5 & $97-138$ & .678 & \\
\hline Performance IQ & 117 & 10.8 & $93-133$ & 120 & 10.3 & $97-134$ & .671 & 50 \\
\hline Full scale IQ & 118 & 9.7 & 104-139 & 122 & 11.6 & $97-140$ & 10 & .40 \\
\hline $\mathrm{AQ}$ & 14.9 & 7.5 & 7-35 & 35 & 6.3 & & 7.980 & $<.00$ \\
\hline
\end{tabular}

Table 2.

Participants' correct response scores for the comprehension questions.

\section{Condition}

Anomalous Non-anomalous

Mean Dif-

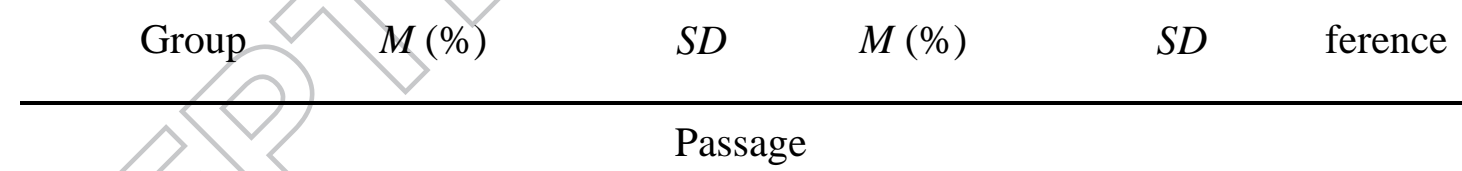

\begin{tabular}{cccccc} 
TD & $9.06(75.50)$ & 2.32 & $11.06(92.17)$ & 0.77 & -2.00 \\
ASD & $8.27(68.92)$ & 1.94 & $10.27(85.58)$ & 1.16 & -2.00 \\
\hline \multicolumn{5}{c}{ Sentence } \\
TD & $8.00(66.67)$ & 2.50 & $8.13(67.75)$ & 2.66 & -0.13 \\
ASD & $7.27(60.58)$ & 2.31 & $7.33(61.08)$ & 2.13 & -0.06
\end{tabular}

Notes. Participants answered 2 questions after each passage of text, there were 6 passage within each condition, therefore each correct response score is out of 12 . 
Table 3.

Descriptive Statistics for Eye Movement Measures in the Critical Word Region.

\section{Condition}

Anomalous Non-anomalous

\begin{tabular}{|c|c|c|c|c|c|c|}
\hline Measure & Group & $M$ & $S D$ & $M$ & $S D$ & Mean Difference \\
\hline \multicolumn{7}{|c|}{ Passage Anomaly } \\
\hline \multirow[t]{2}{*}{ Gaze duration } & TD & 219.42 & 55.18 & 190.58 & 59.36 & \\
\hline & ASD & 230.19 & 82.01 & 188.23 & & 41.96 \\
\hline \multirow[t]{2}{*}{ Regression path } & $\mathrm{TD}$ & 393.90 & 197.76 & 258.49 & & 135.41 \\
\hline & ASD & 330.81 & 147.55 & 440 & 500 & -110.18 \\
\hline \multirow[t]{2}{*}{ Total time } & TD & 374.24 & 126.79 & 78. & 55.30 & 195.92 \\
\hline & ASD & 382.31 & 288.43 & 230.69 & 181.03 & 151.62 \\
\hline \multicolumn{7}{|c|}{ Sentence Anomaly } \\
\hline \multirow[t]{2}{*}{ Gaze duration } & & & 48.16 & 235.9 & 60.21 & 9.77 \\
\hline & & & 79.37 & 231.8 & 53.92 & 26.44 \\
\hline \multirow{4}{*}{ Regression path } & & 329.3 & 135.4 & 326.1 & 113 & 3.14 \\
\hline & ASD & 611.3 & 578.9 & 287.6 & 98.51 & 323.71 \\
\hline & $\mathrm{TD}$ & 390.6 & 118.5 & 254.7 & 61.14 & 135.96 \\
\hline & ASD & 670.3 & 500.8 & 274 & 128.4 & 396.24 \\
\hline
\end{tabular}


Table 4.

Descriptive Statistics for the Eye Movement Measures in the Spill-Over and Passage End Regions.

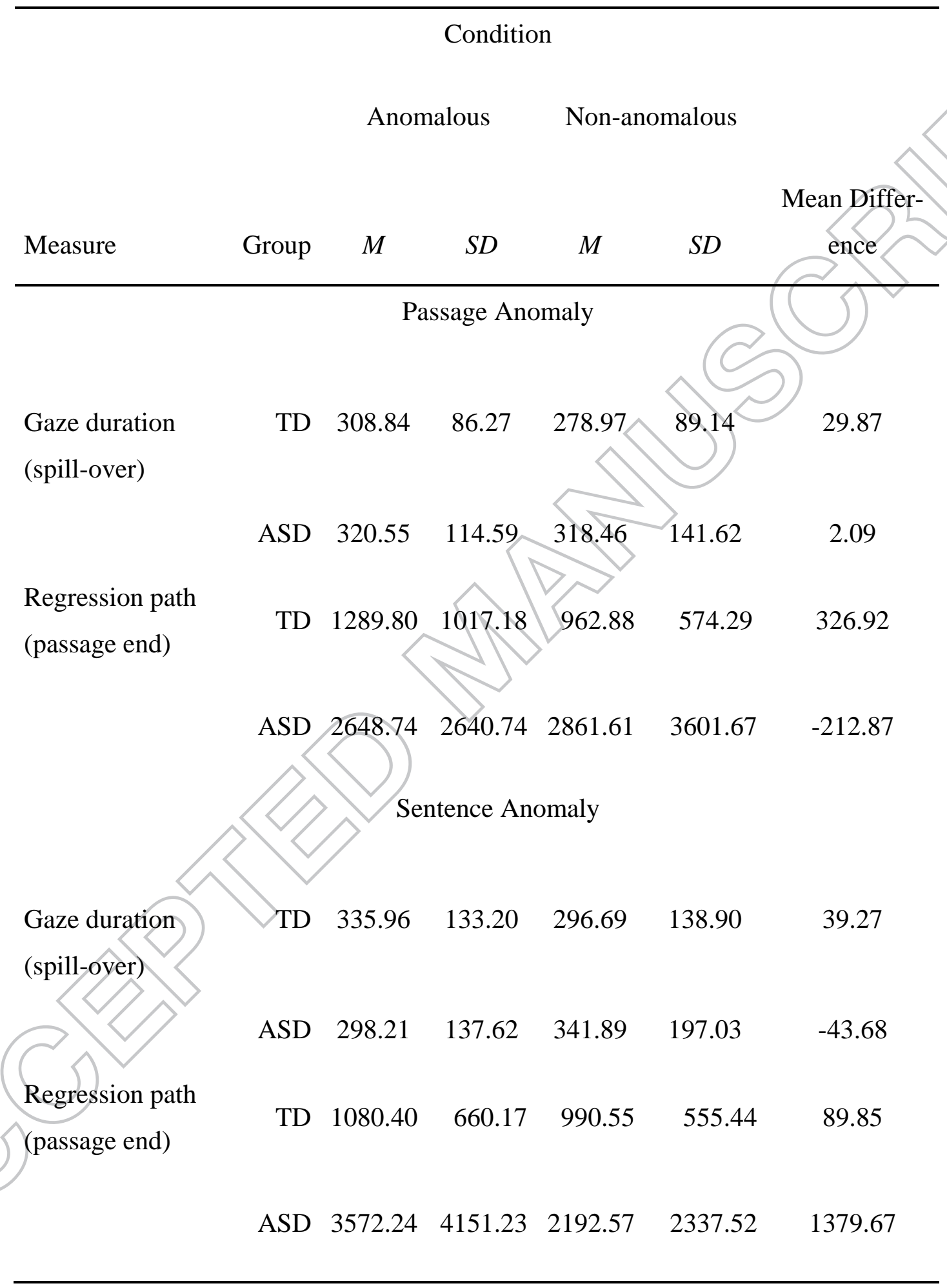


Panel (a)

After three years of hard work on his degree in oriental Studies, scott finally graduated from university. After his graduation, he received a job offer to work in Japan for a year. He was really excited about this and he was planning to take the opportunity to travel around Tokyo, one of the most vibrant cities in East Asia. However, Scott was worried that his inability to speak Japanese/Chinese ${ }^{1}$ would stop $^{2}$ him from communicating with people. He really wanted to be able to make new friends out there ${ }^{3}$.

Panel (b)

There was a tourist flight travelling from Vienna to Barcelona. On the last leg of the journey, it developed engine trouble. Over the Pyrenees, the pilot started to lose control. The plane eventually crashed right on the border. Wreckage was equally strewn in France and Spain. The authorities were trying to decide where to bury the dead/survivors ${ }^{1}$ from the plane ${ }^{2}$ crash. The families of the passengers were devastated about their $\underline{\text { losses }}{ }^{3}$. 


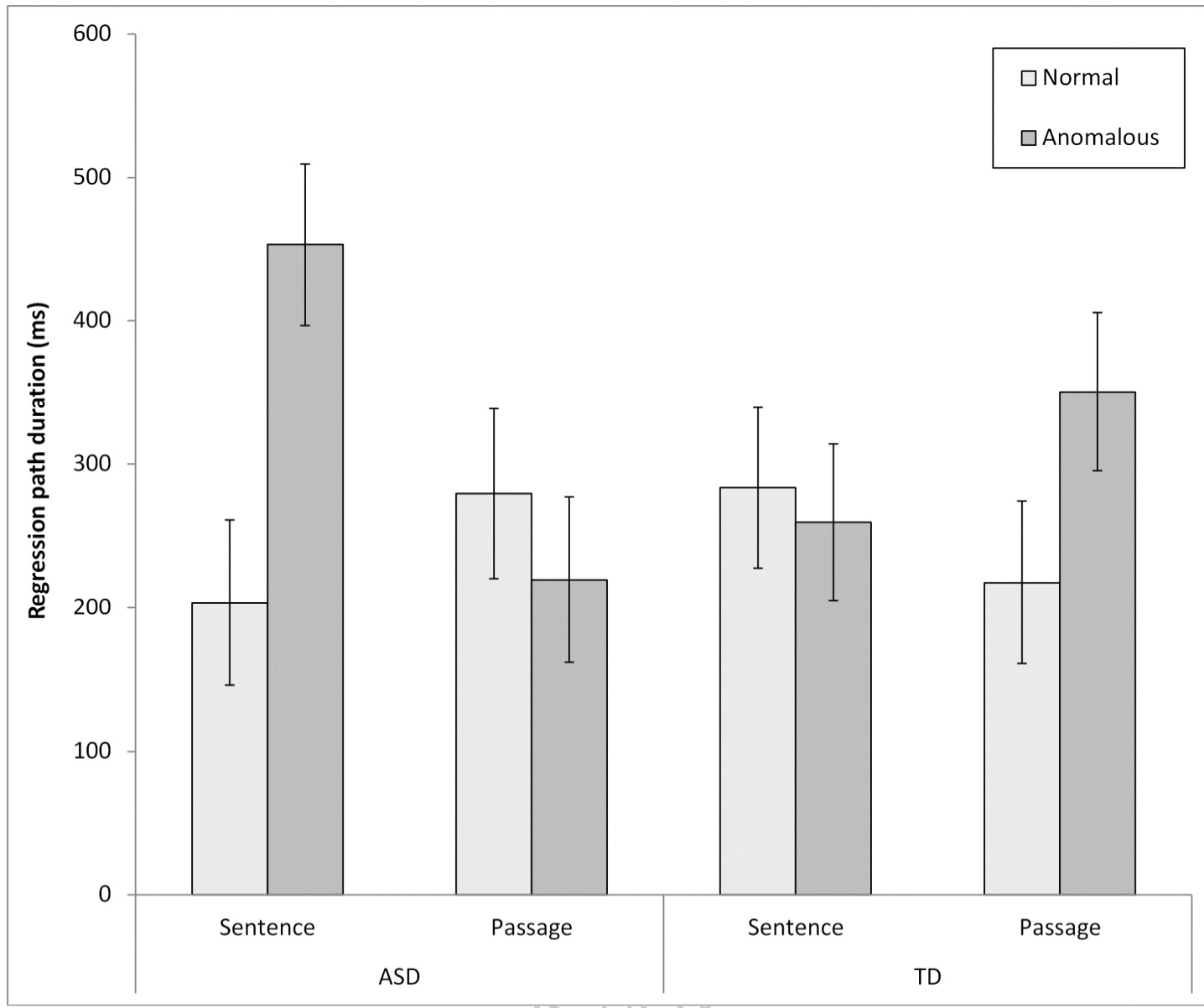

